\title{
El desarrollo local desde una perspectiva sociocultural de la competitividad
}

\author{
Local development from a sociocultural perspective of competitiveness
}

\author{
Le développement local depuis une perspective socio culturelle de compétitivité
}

Rubén D. Echeverry Romero

Profesor Asociado Facultad de Ciencias de la Administración. Grupo de Investigación en Negocios Internacionales y

Comercio Exterior - Categoría B, Universidad del Valle,

Cali - Colombia.

E-mail: ruben.echeverry@correounivalle.edu.co

Javier E. Medina Vásquez

Profesor Titular, Coordinador de la Linea de Investigación en Previsión y Prospectiva del Grupo de Investigación en Previsión y Pensamiento estratégico, Categoria A1, Universidad del Valle, Cali - Colombia. PhD en Ciencias Sociales, Pontificia Universidad Gregoriana,

Roma - Italia.

E-mail: javier.medina@correounivalle.edu.co

\begin{abstract}
Tulio F. Silva Castellanos
Profesor Auxiliar, Grupo de Investigación en Negocios Internacionales y Comercio Exterior - Categoría B, Universidad del Valle, Cali - Colombia. Magister en Ciencias de la Organización, Universidad del Valle, Cali - Colombia. E-mail: tulio.silva@correounivalle.edu.co
\end{abstract}

Artículo de investigación científica y tecnológica según clasificación COLCIENCIAS

Recepción: 20/02/2013

Corrección: 08/04/2013

Aprobación: 07/06/2013

\section{Resumen}

El presente artículo sintetiza una perspectiva sociocultural de la competitividad, como resultado del trabajo de investigación aplicada en ejercicios de asesoría a gobiernos municipales, específicamente en Palmira y Buenaventura que hizo el Grupo de Investigación en Negocios Internacionales y Comercio Exterior, en alianza con el Instituto de Prospectiva, Innovación y Gestión del Conocimiento de la Universidad del Valle. Esta perspectiva se materializa en un esquema metodológico que parte de la realización de un perfil competitivo regional, identifica núcleos temáticos que facilitan la profundización de las problemáticas y el tratamiento de las mismas y define un escenario óptimo para la construcción consensuada de estrategias de desarrollo. En su aplicación ha sido posible comprobar que tanto los núcleos temáticos como las categorías de los problemas identificados resultaron comunes en ambos municipios en sus componentes más genéricos. A pesar del carácter heterogéneo de las experiencias, ha sido posible formular un conjunto de estrategias indispensables en las agendas de desarrollo local para municipios similares en los países en desarrollo. Este artículo constituye un aporte fundamental al conjunto de experiencias de construcción de procesos de desarrollo regional con base en el reconocimiento de las particularidades de una localidad.

Palabras clave: prospectiva, competitividad, desarrollo, región, sociocultural.

\begin{abstract}
This article summarizes a sociocultural perspective of competitiveness as a result of applied work performed by the Research Group in International Business and Foreign Trade, in partnership with the Foresight, Innovation, and Knowledge Management Institute at Universidad del Valle, in consultation exercises for municipal governments, specifically in Palmira and Buenaventura (Colombia). This perspective becomes practical in a methodology that begins with the creation of a regional competitive profile, continues with the definition of thematic groups identified to facilitate further delving into and treatment of regional competitiveness problems and defines an optimal setting for consensus building strategies. In its application, it has been possible to verify that the theme and types of problems identified were generically common in both municipalities. Despite the heterogeneous nature of the experiences, it was possible to formulate a set of indispensable strategies in the Local Development Agendas for similar municipalities in developing countries. This article is a fundamental contribution to the whole experience of building regional development processes based on recognizing the particular characteristics of the location.
\end{abstract}




\section{Résumée}

Cet article synthétise une perspective socio culturelle de la compétitivité, résultat du travail de recherche appliquée sur des exercices de conseil aux gouvernements des municipalités, spécifiquement à Palmira et à Buenaventura, développée par le Groupe de Recherche en Affaires Internationales et Commerce Extérieur, en alliance avec l'Institut de Prospective, Innovation et Gestion de la Connaissance de l'Université del Valle. Cette perspective se matérialise sur un schéma méthodologique qui part de la réalisation d’un profil compétitif régional, qui identifie les noyaux thématiques qui favorisent l'approfondissement des problématiques et le traitement de celles-ci, et définit un scénario optimal pour la construction consensuelle de stratégies de développement. Avec son application, il a été possible de vérifier que tant les noyaux thématiques comme les catégories des problèmes identifiés ont été communs pour les deux municipalités dans ses composants plus génériques. Malgré le caractère hétérogène des expériences, il a été possible de formuler un ensemble de stratégies indispensables pour les agendas de développement local des municipalités similaires dans le pays en développement. Cet article constitue un apport fondamental pour l'ensemble d'expériences de construction de processus de développement régional sur la base de la reconnaissance des particularités d’une localité.

Mots clef: prospective, la compétitivité, le développement, la région, socio-culturel.

\section{Introducción}

Se sintetizan los resultados de la investigación aplicada realizada por el Grupo de Investigación en Negocios Internacionales y Comercio Exterior, en asocio con el Instituto de Prospectiva, Innovación y Gestión del Conocimiento, materializados en la construcción de una síntesis conceptual en torno a la competitividad regional, desde una perspectiva sociocultural, la estructuración de una metodología consecuente con esta apropiación teórica y su aplicación en dos municipios: Palmira y Buenaventura, Colombia.

El propósito fundamental en estos procesos de intervención a la realidad competitiva regional ha sido el de contrastar los avances conceptuales en materia de la perspectiva sociocultural que se ha venido construyendo a lo largo de los años de trabajo de los actores mencionados, al tiempo que esto se pueda materializar en una metodología aplicable y replicable con resultados prácticos y de alto impacto en el desarrollo local.

En la primera parte del artículo, el lector podrá encontrar una síntesis conceptual que sirve de base para la configuración de la metodología. En esta síntesis se destacan las consideraciones fundamentales de la perspectiva que se intenta formular. Es necesario recalcar que a pesar del tiempo que se ha tomado para la construcción de esta propuesta, aún es mucho el camino por allanar, por lo que estos planteamientos son susceptibles de discusión y complemento, tareas que necesariamente contribuirán a la maduración de esta perspectiva.

Consecuente con lo anterior, se formula la metodología que hizo posible la aplicación práctica en cinco procesos divididos en dos grandes etapas. Una primera etapa fundamental para el establecimiento de un marco de referencia necesario para las discusiones posteriores, tanto en materia del diagnóstico o perfil competitivo, como en la selección y análisis de experiencias regionales de similar propósito.

Luego se exponen los resultados del trabajo que se realizó entre los años 2009 y 2011 en ambas localidades, mostrando avances más significativos en Palmira, primer municipio en iniciar este proceso. Estos resultados se presentan de acuerdo con las dos grandes fases del proceso: la definición de los núcleos temáticos, consecuencia del diagnóstico y las primeras aproximaciones a los stakeholders de la competitividad regional y las problemáticas o tendencias pesadas que limitan el desempeño competitivo de ambas regiones y que se exponen en genérico, porque difieren en particularidades inherentes a cada municipio, aunque tienen unidad conceptual.

Con estos resultados, se exponen unas conclusiones en las que se anticipan estrategias que pretenden incorporar la integralidad de las problemáticas enunciadas y que corresponderían a temas indispensables en cualquier agenda de desarrollo local.

En la actualidad, estos proyectos están en manos de los interesados, que ya se encuentran elaborando compromisos de largo aliento para la materialización de los propósitos. El detalle de las experiencias se encuentra en proceso de publicación por parte del Instituto de Prospectiva, Innovación y Gestión del Conocimiento de la Universidad del Valle y se suma como aporte al conjunto de experiencias regionales tan necesario como referente para ejercicios de este mismo tipo para los municipios colombianos y latinoamericanos, en general.

\section{Síntesis conceptual}

Desde finales del siglo XX, la preocupación por entender la competitividad en toda su complejidad y, más aún, por incorporar este conocimiento en procesos deliberados de desarrollo a nivel empresarial, sectorial, regional y nacional, ha derivado en la configuración de planes estratégicos, políticas u otras figuras vinculadas con la planificación que han ido incrementando el acervo de experiencias que en sus éxitos y fracasos terminan por contribuir a las etapas siguientes del mismo proceso.

En Colombia, las reflexiones sobre el tema empiezan a materializarse en la última década del siglo XX, alcanzando su máximo nivel de concreción con la configuración de la Política Nacional para la Productividad y la Competitividad en el año 1999 y construyendo un marco institucional que a través de la red Colombia Compite, pretendió la ejecución de programas ambiciosos entre los que se destaca el apoyo para la configuración de 
cadenas productivas y el Plan Estratégico Exportador 1999-2009, entre otros.

Con cierta periodicidad se realizó seguimiento a los propósitos estratégicos de estas políticas y se impulsó la regionalización del esfuerzo, tema en el que el Valle del Cauca ha sido un referente fundamental a partir de los avances diagnósticos que se han hecho desde la Comisión Regional de Competitividad y la definición de la Agenda Interna del Valle del Cauca en el año 2007 que ha derivado, entre otras cosas, en la identificación de 22 sectores con potencial de desarrollo productivo en el tiempo comprendido para esta Agenda y la identificación de seis ejes temáticos fundamentales para la configuración de un escenario favorable para la creación de ventajas competitivas.

En el año 2008, el tema de la competitividad deja de estar a nivel de una Consejería Presidencial y pasa a tener su propia configuración de Sistema Nacional para la Competitividad y la Productividad, con un fundamento claro en la transformación productiva y la innovación, con una visión renovada para el año 2032.

Tras este recorrido, la experiencia ha dejado tres lecciones que conviene tener presente para la tarea que actualmente se continúa adelantando a nivel regional, y en especial a nivel local:

* La necesidad de profundizar el conocimiento de los sistemas económicos regionales para identificar tanto las actividades que determinan sus perfiles productivos, como aquellas características condicionantes de la evolución de estas actividades.

* La necesidad de promover iniciativas que propendan por la articulación de estas actividades productivas en encadenamientos de generación de valor, que redunden en beneficio de la comunidad inmediata en el corto plazo e impacten el desarrollo regional y nacional en el mediano y largo plazo.

* La necesidad de confeccionar mecanismos de participación público-privada que con base en propósitos estratégicos identificables faciliten la cooperación para la configuración de sinergias cognitivas (Boisier, 2001).

Como producto derivado de estas reflexiones, el Grupo de Investigación en Negocios Internacionales y Comercio Exterior, socio estratégico del Instituto de Prospectiva, Innovación y Gestión del Conocimiento, ambos de la Universidad del Valle, iniciaron en el año 2009 ejercicios que permitieron profundizar diagnósticos locales en experiencias de construcción de Agendas de Competitividad para las ciudades de Palmira y Buenaventura y la construcción de la visión Cali 2036 para la capital vallecaucana.

Una primera actividad que ocupó a los académicos vinculados a estos proyectos fue la construcción de un marco conceptual mínimo para abordar las particularidades de los sistemas competitivos que se analizaron para estas tres regiones. El barrido bibliográfico incluyó inicialmente los autores pioneros en el tema que trabajaron el concepto de competitividad regional, como el caso de Michael Porter, y se perfeccionó con la inclusión de perspectivas más contemporáneas e integradoras como es el caso de la competitividad sistémica, que redefinió los factores determinantes configurando mejor un sistema de interacción de actores que en diferentes niveles realizaban propuestas, proyectos y políticas que de manera articulada impactaban la competitividad de una región (Esser, 1999).

También la incorporación de planteamientos alternativos derivados de la amplia crítica a las formulaciones iniciales de Porter y de las particularidades de las regiones en América Latina y específicamente en Colombia, permitieron establecer que, en primer lugar, la adopción de una visión de desarrollo fundamentada en la competitividad implica un proceso de transformación cultural complejo y de largo plazo, que renueva la perspectiva del mundo y facilita la participación activa de los agentes de desarrollo en la definición de un futuro posible. En segundo lugar, que estos procesos de transformación cultural encuentran en el sujeto regional el escenario concreto para la materialización de este tipo de visión, dadas unas constantes socioculturales que constituyen insumo básico para el consenso (Medina, 1998).

Es decir, en la medida en que la sumatoria de fuerzas generada por los stake holders de la competitividad, tiene particularidades distintas en cada región, se hace necesario construir esquemas metodológicos adaptables a cada caso, de manera que se complementa el ejercicio conceptual con una recomendación para los países en desarrollo, que tienen en común bajos valores para los atributos iniciales que determinan el ambiente competitivo, que es la referencia obligada de casos excepcionales de regiones similares que han logrado esquemas de planeación exitosos, tanto para su desempeño competitivo como para la planificación prospectiva del desarrollo territorial (Barkley, 2008).

A mediados de la primera década del siglo XXI, estas reflexiones fueron incorporadas a las experiencias a nivel sectorial que el Grupo de Investigación en Negocios Internacionales y Comercio Exterior había desarrollado en el contexto de su quehacer académico, obteniendo como resultado una síntesis de los planteamientos críticos mencionados y el enfoque de la CEPAL propuesto a principios de la década de 1990. En esta síntesis se ha hecho el ejercicio de proponer una visión sociocultural de la competitividad, que toma su base en la consideración del sujeto región como un espacio geográfico en constante proceso de definición, cuya autodeterminación es la resultante de la interacción de factores geoeconómicos, institucionales e ideológicos. Esto, corresponde a una interpretación analógica con el esquema metodológico de análisis antropológico de los sistemas socioculturales, cuya evolución puede 
ser explicada a partir del estudio de tres factores determinantes como la ecología, la estructura social y la ideología (Silva, 2004).

Menos que pretender reformular el paradigma competitivo, se hace un aporte que facilita el análisis para la caracterización de un estado actual de la competitividad regional, en especial en cuanto a la descripción de los atributos geoeconómicos e institucionales, reconociendo las particularidades de la región que es objeto de estudio. Esto permite cimentar las bases para la construcción consensuada del desarrollo local más acorde con los intereses de los agentes involucrados (Figura 1).

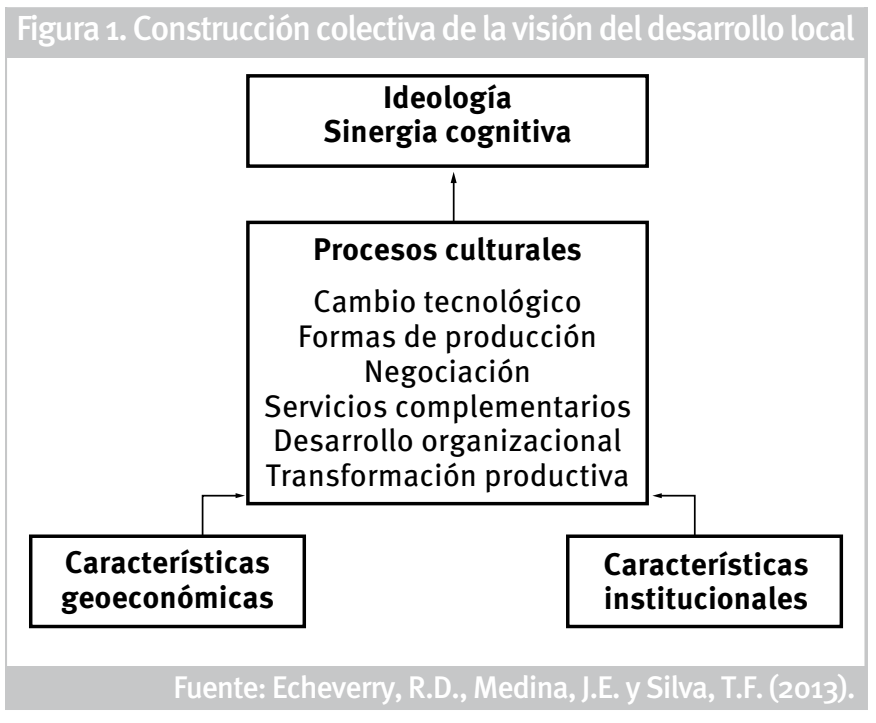

En este enfoque, el análisis geoeconómico permite, por un lado, documentar el conjunto de características que determinan las orientaciones productivas del sujeto regional, distinguiendo en él las maneras de utilización del espacio geográfico y las correspondientes actividades de producción que se llevan a cabo; por el otro lado, permite establecer categorías identificables de espacios geoeconómicos con características más o menos homogéneas que son concomitantes en el sujeto región.

El análisis institucional por su parte, permite identificar los actores en función de sus intereses, lo que constituye el punto de partida para definir el potencial de cooperación para las sinergias cognitivas.

Finalmente, los procesos productivos y los intereses se articulan en un conjunto de núcleos temáticos en torno a los cuales se identifican las problemáticas que han sido limitantes para el desempeño competitivo regional y a partir de ellas, se formulan macroproyectos (proyectos y acciones) que convocan el interés común para la superación de los mismos.

De esta manera, el sistema sociocultural identifica su propio camino al desarrollo a partir del reconocimiento y favorecimiento de procesos culturales que impactan las dimensiones ecológica, institucional e ideológica, como garantes de la evolución de este sistema, de ma- nera que se puedan encausar esfuerzos en el diseño de estrategias favorables a las tendencias y características propias de la región y faciliten la creación de estas sinergias y no la confrontación y la divergencia desarticulante (Echeverry, 2009).

Este enfoque se circunscribe en el contexto de la prospectiva territorial, que concibe el territorio más que como un contenedor de los actores y sus relaciones, como sujeto o actor del desarrollo, que requiere de un proyecto colectivo que lo articule con la nación y de un marco institucional de tipo prospectivo que se resista a la estandarización de las metodologías para el análisis (Medina, 2003).

En rigor, la prospectiva territorial elabora análisis de los sistemas y subsistemas que configuran la región, realiza la exploración de tendencias, define la estrategia que involucra los actores y con la participación de estos, elabora los escenarios probables. Cuando el propósito final es avanzar en la definición de propuestas para la acción, esta secuencia deriva en análisis de probables consecuencias, evaluación de alternativas y cursos de acción recomendados. Este proceso culmina proponiendo opciones teóricas de ordenamiento urbano y territorial cuya intención es corregir las consecuencias no deseadas de la configuración que el escenario referencial anticipa como la más probable (Soms, 2007).

Sin embargo, para el caso particular de las ciudades intermedias, las dificultades que plantean los desorganizados procesos de expansión territorial, el carácter tradicional de sus estructuras socioproductivas y en general, la poca disponibilidad y calidad de la información imposibilitan un ejercicio de esta magnitud. A pesar de todo, lo que en la práctica se ha podido determinar a partir de la experiencia que se documenta en el presente trabajo, permite suponer que tanto el esquema de análisis como los resultados obtenidos constituyen un referente importante para que regiones similares encuentren el camino endógeno hacia el mejoramiento de sus condiciones competitivas.

\section{Metodología}

El estudio que permitió aplicar esta perspectiva se aplicó inicialmente en el desarrollo del diagnóstico del eje tecnoeconómico para la Visión Cali 2036, un análisis previo que pondría en discusión las tendencias pesadas que impactan el desempeño competitivo de la capital vallecaucana. No obstante, la mayor oportunidad para su aplicación y seguimiento se presentó en la construcción de las Agendas de Competitividad para Palmira y Buenaventura, los dos municipios más importantes del Valle del Cauca, después de su capital, en términos de aporte económico y demográfico.

En el desarrollo del trabajo se destacan cinco actividades fundamentales que se sintetizan en el Figura 2. 


\section{Figura 2. Proceso de construcción de las agendas de desarrollo local}

\begin{tabular}{|c|c|c|c|c|}
\hline $\begin{array}{l}\text { Perfil competitivo } \\
\text { Caracterización de } \\
\text { potencialidades y } \\
\text { limitaciones }\end{array}$ & $\begin{array}{c}\text { Marco de referencia } \\
\text { Definición de términos } \\
\text { de referencia y } \\
\text { metodología para el } \\
\text { consumo }\end{array}$ & $\begin{array}{c}\text { Formulación de } \\
\text { propuestas } \\
\text { Exploración para } \\
\text { identificar iniciativas de } \\
\text { mejoramiento de } \\
\text { condiciones competitivas }\end{array}$ & $\begin{array}{c}\text { Definición de } \\
\text { prioridades } \\
\text { Establecimiento de } \\
\text { condiciones de base y } \\
\text { priorización de proyectos } \\
\text { y sectores productivos }\end{array}$ & $\begin{array}{c}\text { Legitimación de la } \\
\text { propuesta } \\
\text { Definición de los } \\
\text { instrumentos para } \\
\text { convertir la propuesta en } \\
\text { una carta de navegación } \\
\text { para el desarrollo }\end{array}$ \\
\hline
\end{tabular}

Fuente: Echeverry, R.D., Medina, J.E. y Silva, T.F. (2013).

Este esquema metodológico se puede explicar de la siguiente manera:

* Perfil competitivo regional. Este proceso inicial permite acopiar información primaria y secundaria en fuentes documentales de diferentes autoridades municipales y regionales, de manera que se pueda obtener una visión panorámica de la problemática competitiva de la localidad, punto inicial que a la vez que sirve de ilustración, permite orientar la discusión. Esto es importante porque en muchos casos se puede encontrar un aparente exceso de diagnósticos en diferentes áreas, lo que pudo percibirse en los casos que han sido objeto de análisis en el trabajo que aquí se presenta, pero lo que en realidad existe es dispersión de información y falta de claridad en el paradigma de desarrollo que servirá como referencia para el ejercicio de proyección. En esta etapa, la revisión documental retroalimenta sesiones que con la metodología de foros de discusión permiten precisar elementos diagnósticos y ser positivos en la formulación de las particularidades de la problemática.

* Definición del marco de referencia. Acompaña la identificación de las problemáticas más importantes, la búsqueda calificada de experiencias regionales que en el país y en el mundo han identificado sus hojas de ruta para el desarrollo productivo y presentan similitudes con ambos municipios. A través de un ejercicio bibliométrico se logró establecer como referentes fundamentales a San Luis Potosí (México) y Valencia (España) para el caso de Palmira, y a Guayaquil (Ecuador), Colón (Panamá), Santos (Brasil), Buenos Aires (Argentina), Cartagena (Colombia), Manzanillo (México), Callao (Perú), entre otros, para el caso de Buenaventura.

* Formulación de propuestas. Constituye el principal espacio de deliberación. Como trabajo preliminar, los hallazgos del diagnóstico y del marco de referencia permiten un acercamiento a los actores interesados para definir con ellos una metodología de trabajo para garantizar la unidad temática de las discusiones. Este trabajo permitió identificar comúnmente seis núcleos temáticos que se articulan en los aspectos geoeconómicos e institucionales, como se muestra más adelante. Es la etapa más prolongada y participativa del trabajo y se desarrolló en cuatro meses en cada caso, garantizando la convocatoria abierta a todos los miembros de la comunidad.

* Definición de prioridades. Toma como insumo fundamental las propuestas identificadas en materia de sectores y proyectos que por su carácter incluyente y articulante en cuanto a lo productivo, son susceptibles de prioridad. Esta relación se somete a criterio de expertos que definen una primera aproximación a la factibilidad e importancia de los proyectos y se corresponde con la estructuración de la Agenda que se presentará de nuevo a la comunidad interesada.

* Legitimación de la propuesta. Constituye la presentación en sociedad de la Agenda de Competitividad y el ejercicio de compromiso de los actores que manifiestan sus voluntades a través de la adopción de un Gran Pacto Colectivo que le confiere a la Agenda el carácter de política pública para el desarrollo productivo fundamentado en la competitividad.

Este ejercicio se favorece con la integración de los actores socioeconómicos más representativos de los diferentes sectores de la comunidad, de manera que el liderazgo y la capacidad de convocatoria son condiciones fundamentales para garantizar la legitimidad en sus resultados. De ahí que esta integración debe reconocer actores sociales de diferentes sectores (productivo, gubernamental, académico, etc.).

Para el caso específico de Palmira y Buenaventura, la metodología comprendió dos grandes fases basadas en las consideraciones anteriores. En la primera fase predominó el establecimiento de una primera aproximación al perfil competitivo del municipio y la definición de un marco de referencia mínimo; la contrastación de los resultados preliminares de esta fase permitió identificar los actores involucrados, establecer objetivos concretos y definir una metodología de trabajo adecuada para mantener focalizada la discusión. De manera más específica, el perfil competitivo además de brindar un panorama general sobre el estado de la competitividad de estas dos ciudades, fue a su vez, insumo fundamental para la definición de los núcleos temáticos que orientaron las discusiones que, en una primera instancia permitieron 
mayor precisión en la caracterización de cada problema, y lo más importante, una mayor riqueza en cuanto a la formulación de propuestas para su superación, tarea en la que se hizo énfasis en la segunda etapa del trabajo.

La identificación de los actores institucionales permitió garantizar la idoneidad y la representatividad, base para el establecimiento de compromisos de largo aliento y de legitimidad tanto en la definición del escenario deseado como en los instrumentos políticos que se aplicarán para hacer efectivos los logros planteados.

La segunda fase del proyecto, como ya se mencionó, se concentró en lo propositivo. Los mencionados núcleos temáticos que se lograron definir a partir del perfeccionamiento del diagnóstico, contribuyeron a focalizar la participación de los actores de acuerdo con su conocimiento en las temáticas específicas, para garantizar un efecto de concreción en las conclusiones. De otro lado, la regularidad en las sesiones de trabajo, que se respetó con rigurosidad, mantuvo el interés de los convocados y garantizó la continuidad en el debate.

Una vez identificados los proyectos críticos para el mejoramiento de las condiciones competitivas identificadas, los resultados se sometieron a conceptos especializados que centraron sus apreciaciones en materia de factibilidad y prioridad de los mismos.

De esta manera se procuró garantizar a lo largo del proceso la permanencia de los elementos fundamentales que han representado el éxito en otras experiencias revisadas como la voluntad política de las autoridades locales, la articulación institucional de los actores claves, los focos temáticos bien definidos, el nivel de especialización e inclusión de los procedimientos utilizados y la capacidad técnica (competencias) de los agentes involucrados, y la preocupación por vincular líderes sociales capaces de transmitir el mensaje y coordinar el proceso, lo que garantiza la legitimidad del trabajo y, por supuesto, la institucionalización del mismo.

\section{Resultados y discusión}

Los principales resultados de la aplicación de esta metodología han quedado expuestos en sendas publicaciones que a la fecha de postulación del presente artículo se encuentran en proceso de edición y tratan en extenso los detalles de cada ejercicio. Sin embargo, se pueden sintetizar en dos categorías: la identificación de los ejes temáticos y las principales características del sistema competitivo de las localidades (tendencias pesadas).

Los núcleos temáticos corresponden al término de la primera fase del proyecto y con base en el perfil competitivo, ayudan a guiar las discusiones de las mesas de trabajo. En cada uno de ellos se coordinan subtemas así:

* Desarrollo empresarial y sectores estratégicos. En este núcleo temático se abordaron los temas relacionados con el perfil productivo de las empresas de la región, las barreras existentes para la integración empresarial, los mecanismos para la promoción de la actividad empresarial y la inversión y definición de sectores estratégicos para el desarrollo de su potencial en el corto, mediano y largo plazo.

* Infraestructura y logística. La agenda de este núcleo incluyó todo lo concerniente a la dotación física y de servicios que facilita la instalación de unidades productivas y habitacionales, así como la movilidad urbana y rural, la conectividad vial y los servicios básicos, incluyendo el estado actual de las TIC elementos.

* Desarrollo territorial. Aquí se incorporaron los temas relacionados con la planificación del desarrollo territorial, los proyectos en marcha y potenciales para la dotación de infraestructura, desarrollo de actividades productivas y de vivienda. La sostenibilidad del desarrollo constituyó el eje fundamental de las discusiones que se complementaron con temas relacionados con la conservación de los recursos hídricos y forestales, y con la reducción del impacto negativo de la actividad empresarial en el medio ambiente.

* Ciencia, tecnología e innovación. Correspondió a este núcleo temático el análisis de las condiciones actuales para la creación de conocimiento y su incorporación en procesos de innovación con impacto en el sector productivo. Se analizó el papel de las universidades y centros de investigación, así como de otras empresas que presentan experiencias vigentes e importantes en el tema.

* Desarrollo social. A pesar de la complejidad del tema, este núcleo temático privilegió los aspectos relacionados con la calidad y cobertura en educación y salud y se incorporaron temas sensibles en materia de seguridad, vivienda y problemáticas específicas de las minorías. La especialidad de cada tema planteó una exigencia en el perfil de los participantes, sobre todo a nivel de las ponencias, en las que se privilegió el componente propositivo.

* Concertación e integración sector público-sector privado. Abordó el análisis del mapa institucional y de los mecanismos formales para la integración interinstitucional y la necesidad de legitimar el ejercicio que plantea la Agenda de Competitividad. Este constituyó el núcleo temático importante para la determinación de la articulación para las sinergias cognitivas, de las que se ha hablado en párrafos anteriores.

Con base en estas categorías, el perfeccionamiento del diagnóstico permitió realizar una síntesis de las condiciones de base para la competitividad. Vale la pena aclarar que, por las restricciones de espacio para este artículo, se relaciona a continuación una síntesis de los principales hallazgos, dejando la caracterización en extenso para posteriores publicaciones que se referirán de 
manera exclusiva a cada experiencia, con la respectiva documentación.

Problemáticas persistentes desde el punto de vista geoeconómico:

* Desarrollo empresarial y sectores estratégicos. En ambas regiones predominan empresas micro y pequeñas en poco más del $90 \%$, las medianas componen alrededor del $8 \%$ y las grandes son apenas un mínimo del total. No obstante, la contribución a la producción de estas últimas es la mayor, superando el $60 \%$ en cada caso. También predomina la concentración de un solo sector que está desarticulado del resto de las actividades productivas, como es el caso del comercio, que contiene una heterogénea variedad de negocios muy riesgosos, poco rentables y con períodos de vida inferiores a 5 años. Llama la atención en ambos casos que los sectores por los que tradicionalmente son conocidas estas regiones (actividades portuarias para el caso de Buenaventura y agroindustria del azúcar en el caso de Palmira) distan mucho de liderar procesos de integración con los demás sectores productivos. Finalmente, el estilo gerencial asociado con las empresas predominantes permite pensar en una administración por contingencias, poco calificada, con escasa visión estratégica y una orientación hacia el ostracismo.

* Infraestructura y logística. La debilidad endémica para la competitividad regional es la infraestructura en todo el territorio nacional. Si bien la movilidad interna es común en ambas ciudades, la interconexión con el resto del país presenta un panorama mucho mejor para Palmira que para Buenaventura, cuando debería ser al contrario, por su papel estratégico para el comercio exterior colombiano. No obstante, la íntima relación entre estas dos ciudades, una por ser puerto y otra por ser punto nodular para la conectividad del comercio con el resto del país, genera oportunidades al considerar la posibilidad de convertir a Palmira en una zona logística integral, lo que sin duda genera alternativas para descongestionar el puerto. En cualquiera de estos escenarios, la clave está en el urgente mejoramiento de la infraestructura vial. Tema aparte constituye la dotación mínima de servicios para el desarrollo empresarial que, en las zonas que han sido dispuestas para el desarrollo de actividades que no son exclusivamente comercio, se presentan serias deficiencias, sobre todo en lo relacionado con la dotación de gas como fuente alternativa de energía $y$ telecomunicaciones. El caso del acueducto y alcantarillado es particularmente crítico para Buenaventura.

* Desarrollo territorial. En cada uno de los casos se encontraron subregiones con grados de desarrollo socioeconómico y dotación de infraestructura disímiles al resto de la población. En Buenaventura se identifican cuatro subregiones: la zona portuaria, la zona urbana (con predominio del comercio y residencias), la interconexión con el litoral (con predominio de poblaciones marginales con escasos medios de comunicación y transporte) y la interconexión vial con el interior (con predominio de actividades de explotación indiscriminada del suelo). En el caso de Palmira, se diferencia la zona urbana (con predominio de actividades comerciales y residenciales), la zona de ladera (con predominio de fincas de recreo y agricultura en pequeña escala) y la zona de conexión con Cali (con un fuerte predominio industrial). En todos los casos, la ausencia de planificación ha afectado el criterio de uso del suelo con criterios de sustentabilidad y el desmedido crecimiento de la zona urbana (menos del $2 \%$ del territorio en cada caso) ha concentrado las actividades en un breve espacio territorial promoviendo problemáticas típicas del hacinamiento (movilidad, salubridad, seguridad, etc.) y abandono de las áreas rurales.

Problemáticas persistentes desde el punto de vista institucional:

* Ciencia, tecnología e innovación. En este factor se encuentran claras distinciones en ambas regiones. En Palmira, el potencial para el desarrollo de ventajas en estos tópicos es importante. Existen 9 universidades y 4 centros de investigación reconocidos mundialmente, en ambos casos, con una clara orientación de la actividad investigativa hacia la agroindustria. Buenaventura, por su parte, cuenta con el Centro Náutico Pesquero del SENA, y a pesar de que otras 4 instituciones de educación superior atienden su demanda formativa, la desarticulación entre ellas para proyectos de investigación es evidente. A esto se adiciona el perfil de las empresas de la región, que con un predominio de orientación hacia el comercio, el impacto de la investigación para la innovación y la transformación productiva, es bastante limitado y no se compadece con la realidad predominante.

* Desarrollo social. En este tema también hay claras diferencias. Mientras Palmira enfrenta problemas en materia de calidad de educación en todos los niveles, salud y seguridad, Buenaventura adiciona a estos problemas un matiz crítico en materia de cobertura. Palmira tiene al menos la alternativa en Cali, a sólo $30 \mathrm{~km}$ de distancia, pero Buenaventura dista mucho del epicentro de toma de decisiones departamentales. La calidad de vida en el puerto es mínima con un predominio del problema de dotación de servicios de agua y alcantarillado y un creciente dilema en materia de seguridad. Sin embargo, se destaca mayor unidad sociocultural por la solidez de las constantes culturales de esta región, cuando se compara con el interior, lo que representa un componente básico para la transformación sociocultural. 
* Concertación sector público-sector privado. En ambas regiones existe se puede denominar como "infraestructura institucional", es decir, representatividad básica en organismos públicos y privados con intereses identificables, pero sin comunicación entre ellos. La articulación que falta entre estos actores depende en gran medida de la recuperación de la confianza, en especial en las instituciones gubernamentales. Los liderazgos tradicionales han caducado y la renovación está ausente. El caso es más dramático en Buenaventura, donde este abandono ha derivado en procesos de maduración del conflicto armado en todas las subregiones, lo que incrementa la entropía entre las instituciones que no encuentran espacios comunes para el consenso. No obstante, la existencia de comités cívicos intergremiales, con una activa participación de fuerzas vivas de la comunidad, se presenta como una oportunidad que puede ser potencializada a partir del liderazgo cívico y la confianza.

En América Latina se han revisado experiencias en este mismo sentido para casos específicos como Córdoba, Rosario y Buenos Aires en Argentina, Santiago en Chile, Trujillo y Lima en Perú, entre otras, evidenciando que el éxito de este tipo de planificación radica en la voluntad política de las autoridades locales, la articulación institucional de los actores claves, los focos temáticos bien definidos, el nivel de especialización e inclusión de los procedimientos utilizados y la capacidad técnica (competencias) de los agentes involucrados. Por supuesto, la mayor parte de estos aspectos están garantizados por las capacidades de los líderes sociales para comunicar y coordinar el proceso, así como de la institucionalización del mismo (Steinberg, 2005).

De esta manera, el diagnóstico competitivo regional adquiere mucha relevancia en la identificación de las características que la región desea potencializar para mejorar sus condiciones para la creación de ventajas competitivas, identifica actores, sectores, proyectos y tendencias sobre la base de un esquema posible, dejando establecidos los cimientos para que la planificación del ordenamiento territorial sea consecuente con las dinámicas pesadas y las tendencias emergentes que se puedan determinar a partir del estudio de estas potencialidades, como lo requiere el marco institucional de tipo prospectivo.

\section{Conclusiones}

La acumulación de experiencias de construcción consensuada del desarrollo regional es una importante tarea que encuentra en su divulgación la oportunidad de enriquecer los marcos de referencia que reducen las probabilidades de error al poner en conocimiento lo que en otras regiones similares se ha adelantado con respecto al tema de la planificación del desarrollo local con base en la competitividad. El debate que se genera alrededor de este conocimiento socializado contribuye igualmente al perfeccionamiento de metodologías que, como la expuesta en este trabajo pretenden ir más allá de ser ejercicios académicos y convertirse en guías para el establecimiento de políticas de desarrollo en las regiones de Colombia y América Latina en general.

El diagnóstico o perfil competitivo y la retroalimentación del trabajo de las mesas de discusión en torno a los ejes temáticos permite la identificación de estrategias para el mejoramiento competitivo regional, esto es, la configuración de procesos culturales que se articulan en una predisposición ideológica positiva hacia la competitividad, al encontrar legitimidad a través del consenso.

Tanto para el caso de Palmira, como para Buenaventura, se pueden relacionar un conjunto común de estrategias que desde ya se perfilan como componentes genéricos indispensables en una Agenda de Desarrollo Local, a saber:

* Planificación del ordenamiento territorial de largo plazo. Las herramientas políticas actuales son cortoplacistas y poco eficaces para garantizar una solución al problema. De esta manera, la prospectiva territorial se convierte en un ejercicio necesario que permite diagnosticar la utlización del terreno y realizar proyecciones sobre la base de tendencias y proyectos críticos acordes con una visión de desarrollo consensuado, por lo que se presenta como una tarea fundamental que da contexto de desarrollo a una agenda de desarrollo local con base en la competitividad.

* Identificación de macroproyectos que promuevan la integración empresarial. La crisis de liderazgo de los sectores productivos tradicionales en las economías regionales, las nuevas tendencias de consumo y la amenaza permanente de la competencia que proviene de mercados externos, obliga a generar sinergias alrededor de un proyecto que convoque el interés común. Los mecanismos de aglomeración empresarial facilitan la generación de encadenamientos productivos y de redes de cooperación que involucran proveedores, consumidores, instituciones de apoyo y requerimientos de recurso humano calificado y redunda en beneficio de una comunidad que encontrará oportunidades de generación de riqueza a partir de nuevas plazas de trabajo. En el caso de Palmira, la apuesta correspondió al proyecto de crear un parque científico y tecnológico; para el caso de Buenaventura, la apuesta se perfila por un ecoparque industrial con una fuerte orientación hacia las actividades portuarias y la conservación sostenible del litoral, aunque en este caso la formulación de sus condiciones de base están aún por identificarse.

* Definición de las principales apuestas productivas. Es igualmente importante que los macroproyectos de integración se asocien, al menos inicialmente, con sectores productivos con dinámica y participación importante en el sistema productivo regional, de ma- 
nera que se pueda sacar provecho de la experiencia y tradición de estos sectores, como apalancamientos fundamentales para el crecimiento, la atracción de inversiones y de actividades que complementen estos encadenamientos. También es necesario definir agendas sectoriales que reconozcan las particularidades de la dinámica de cada actividad económica, de manera que se identifiquen proyectos particulares que se puedan articular con los propósitos estratégicos de la agenda de competitividad regional, estableciendo claramente requisitos de base para la factibilidad de los mismos.

*Articulación del sector empresarial y la academia. La academia y en general, las instituciones que promuevan la gestión del conocimiento, deben orientar sus esfuerzos a la formación en competencias específicas que reconozcan las exigencias de las principales apuestas regionales, de manera que se garantice mayor oportunidad de inserción laboral de los habitantes propios de la región en la actividad productiva y la generación de ingresos para los mismos. Esta es una tarea fundamental, sobre todo considerando que en este tipo de regiones la educación superior es básicamente ofrecida por instituciones estatales.

* Renovación de los liderazgos tradicionales. Es necesario aclarar que esta misión está obligatoriamente ligada a la formación de los líderes del futuro, que recibirán la posta de los liderazgos tradicionales que se perfilan en declive. En este sentido, la formación que promueven las instituciones de educación superior, no pueden abandonar la tarea de dotar a los individuos de un mínimo arsenal de conocimiento que permita la construcción de una cosmovisión que considere la realidad en su complejidad y al tiempo, incremente las posibilidades de proyectar una visión de desarrollo que convoque legitimidad a través de la integralidad de proyectos viables y no de imágenes caudillistas individuales.

* Gestión y políticas públicas de intervención para la competitividad. Como derivación final de lo expuesto, es necesario señalar que estos planteamientos deben ser consignados en un instrumento de política pública que se legitime a través de los organismos de gobierno locales, de manera que se constituyan en herramientas fundamentales para la planeación del desarrollo regional. En ellos, deben quedar consignados, además, los mecanismos que garanticen la suficiente flexibilidad para adaptar la agenda a los cambios en las tendencias externas y que promuevan la participación permanente e incluyente, así como las alianzas público-privadas, lo que le da el carácter consensuado a la estrategia de desarrollo regional. Por ello, el seguimiento de los programas se convierte en una tarea fundamental y de carácter permanente para dar garantía de éxito a los propósitos planteados en la Agenda de Competitividad Regional.

En consecuencia con el enfoque teórico y metodológico propuesto en este artículo, estos planteamientos se ilustran en el Figura 3.

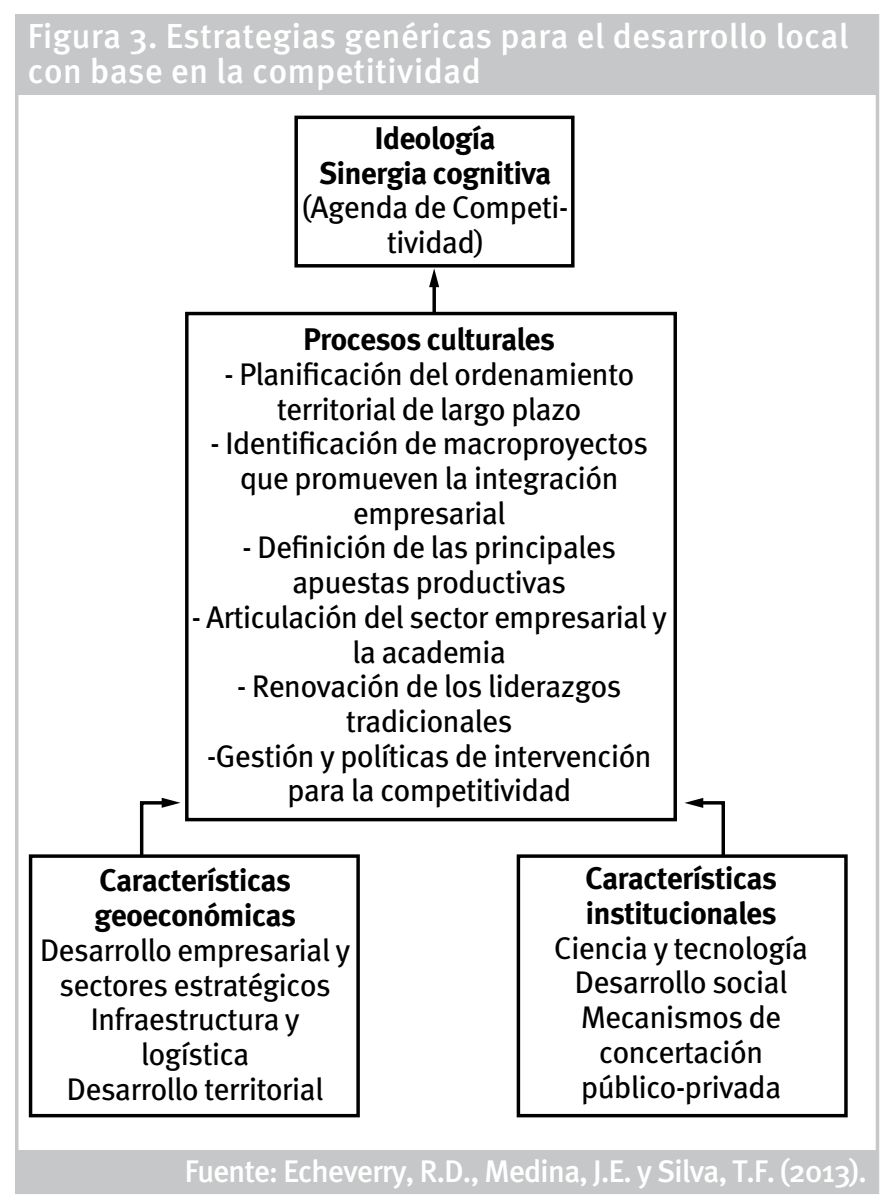

Al final, este ejercicio permite materializar un gran pacto colectivo de carácter permanente como un proyecto de desarrollo, cuya legitimidad se soporta en el consenso que condujo a su construcción, es decir, la construcción de sinergias cognitivas para el desarrollo local. Actualmente, este último paso lo surte la Agenda de Competitividad de Palmira, que ha puesto en conocimiento de las autoridades políticas municipales, los resultados del estudio que se han sintetizado en este artículo y con base en ellos, se propone un Acuerdo Municipal, legitimado por el Concejo Municipal que desde ya ha empezado a permear los planes de desarrollo en los dos últimos períodos de gobierno.

\section{Referencias}

Barkley, D. (2008). Evaluations of regional competitiveness: Making a case for case studies. The Review of Regional Studies, 38 (2) (pp. 121-143).

Boisier, S. (2001). Sociedad del conocimiento, conocimiento social y gestión territorial. Santiago de Chile, Chile: Universidad Católica de Chile. 
Boisier, S. (2003). ¿Y si el desarrollo fuese una emergencia sistémica? Cuadernos de Administración - Universidad del Valle, 19 (29) (pp. 47-79).

Echeverry, R. y Silva, T.F. (2009). Identificación de los principales factores que afectan el desempeño competitivo del subsector avícola en el Valle del Cauca. Pensamiento y Gestión, (27) (pp. 284-318).

Esser, K. (1999). Competencia global y libertad de acción nacional. Nuevo desafío para las empresas, el estado y la sociedad. Caracas, Venezuela: Nueva Sociedad.

Lombana, J. y Rozas, S. (2009). Marco analítico de la competitividad. Fundamentos para el estudio de la competitividad regional. Pensamiento y Gestión, (26) (pp. 1-39).

Medina V, J. (1998). Las visiones de futuro y el pensamiento a largo plazo: fundamentos de la competitividad. En: Ruiz, M.dP., Arenas, M.C. y Medina, J.E. Consejería económica y de competitividad. Ruptura cultural para el desarrollo: memorias de los coloquios colombianos de cultura de la competitividad (pp. 137-184). Cali, Colombia: Universidad del Valle.
Medina V, J. (1997). Los aspectos psicosociales y culturales en la competitividad y la gestión del desarrollo regional. En: Medina, J. y Varela, E. (Compiladores) Globalización y gestión del desarrollo regional. Perspectivas Latinoamericanas (pp. 215-236). Cali, Colombia: Universidad del Valle.

Medina V, J. (2003). Las transformaciones de la prospectiva territorial y la formación de los futuristas: etapas, perfiles y desafíos. Cuadernos de Administración - Universidad del Valle, 19 (29) (pp. 9-45).

Porter, M. (1991). La ventaja competitiva de las naciones. Buenos Aires, Argentina: Javier Vergara (Ed.).

Silva, T. (2004). La competitividad como característica intrínseca de los sistemas socioculturales. Cuadernos de Administración - Universidad del Valle, 20 (32) (pp. 41-66).

Soms, E. (2007). Estudios de competitividad en sistemas Urbano Territoriales. Revista URBANO, 10 (15) (pp. 20-30).

Steinberg, F. (2005). Strategic urban planning in Latin América: Experiences of building and managing the future. Habitat International, 29 (1) (pp. 69-93).

Cuadernos de Administración / Facultad de Ciencias de la Administración / Universidad del Valle Periodicidad: semestral / ISSN impreso No 0120-4645 - ISSN electrónico N²256-5078 / Nombre abreviado: cuad.adm. Edición Vol. $29 \mathrm{~N}^{\circ} 49$ (enero - junio de 2013)

El desarrollo local desde una perspectiva sociocultural de la competitividad/Rubén D. Echeverry Romero, Javier E. Medina Vásquez y Tulio F. Silva Castellanos 